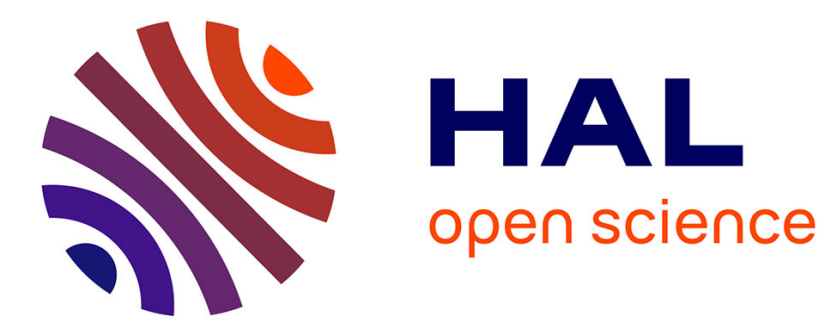

\title{
Adaptive spread spectrum multicarrier multiple-access for UWB systems
}

\author{
Antoine Stephan, Jean-Yves Baudais, Jean-François Hélard
}

\section{To cite this version:}

Antoine Stephan, Jean-Yves Baudais, Jean-François Hélard. Adaptive spread spectrum multicarrier multiple-access for UWB systems. IEEE Vehicular Technology Conference (VTC'07 Spring), Apr 2007, Dublin, Ireland. pp.2926-2930, 10.1109/VETECS.2007.600 . hal-00194979

\section{HAL Id: hal-00194979 \\ https://hal.science/hal-00194979}

Submitted on 7 Dec 2007

HAL is a multi-disciplinary open access archive for the deposit and dissemination of scientific research documents, whether they are published or not. The documents may come from teaching and research institutions in France or abroad, or from public or private research centers.
L'archive ouverte pluridisciplinaire HAL, est destinée au dépôt et à la diffusion de documents scientifiques de niveau recherche, publiés ou non, émanant des établissements d'enseignement et de recherche français ou étrangers, des laboratoires publics ou privés. 


\title{
Adaptive Spread Spectrum Multicarrier Multiple-Access for UWB Systems
}

\author{
Antoine Stephan, Jean-Yves Baudais and Jean-François Hélard \\ Institute of Electronics and Telecommunications of Rennes (IETR) \\ INSA IETR, 20 Avenue des Buttes de Coesmes, 35043 Rennes, France \\ antoine.stephan@ens.insa-rennes.fr, \{jean-yves.baudais ; jean-francois.helard\}@insa-rennes.fr
}

\begin{abstract}
Ultra-wideband (UWB) has emerged as an exciting technology for short range, high data rate wireless personal area networks (WPAN) applications. The multiband orthogonal frequency division multiplexing (MB-OFDM) scheme is one of the main candidates for WPAN standardization. In this paper, we propose a new UWB waveform based on a spread spectrum multicarrier multiple-access (SS-MC-MA) scheme that respects the OFDM parameters of the MB-OFDM solution. Then, we suggest an efficient low-complexity resource allocation algorithm that maximizes the total system throughput. Furthermore, we dynamically distribute the time-frequency codes that provide frequency hopping between users, in order to improve our system performance. We show that our proposed system transmits data at much higher attenuation levels and higher throughputs compared to the MB-OFDM system. Consequently, we conclude that our proposed system can be advantageously exploited for high data rate UWB applications.
\end{abstract}

Keywords-Multicarrier CDMA, resource allocation, spread spectrum multicarrier multiple-access (SS-MC-MA), ultrawideband (UWB), wireless access.

\section{INTRODUCTION}

Ultra-wideband (UWB) technology has attracted significant interest in wireless communications since 2002 when the Federal Communications Commission (FCC) agreed on the allocation of a $3.1-10.6 \mathrm{GHz}$ spectrum for unlicensed use of UWB devices [1]. The FCC regulations are very stringent since UWB spectrum overlays other existing spectrum allocations. In order to reduce interference with these existing services, the FCC imposed a power spectral density (PSD) limit of -41.25 $\mathrm{dBm} / \mathrm{MHz}$.

The IEEE 802.15.3a wireless personal area networks (WPAN) standardization group proposed a very high data rate physical layer based on UWB signaling [2], since UWB can provide high data rate at low cost and relatively low power consumption. The main multiple-access techniques considered by the group are a pulse radio transmission which uses directsequence code-division multiple-access (DS-CDMA), and a multiband orthogonal frequency division multiplexing (MBOFDM) supported by the Multiband OFDM Alliance (MBOA) [3]. The MBOA solution appears to be a very competitive candidate due to its ability to mitigate interference and to achieve high data rate. Other techniques based on a multicarrier code-division multiple-access (MC-CDMA) scheme have also been proposed in the literature in order to reach much higher data rate [4].

In the UWB environment, the channel response varies slowly in time and can be considered as quasi-static. Previous resource allocation studies have been proposed for the MBOA model [5]. In this paper, we propose a new UWB waveform based on a spread spectrum multicarrier multiple-access (SSMC-MA) scheme [6] respecting the OFDM parameters of the MBOA solution, and we propose an efficient low-complexity resource allocation algorithm applied to the SS-MC-MA scheme in order to maximize the total system throughput, while respecting the FCC regulations. Furthermore, we propose a new technique that maximizes the throughput by dynamically distributing the time-frequency codes that provide frequency hopping between users.

This paper is organized as follows. Section II presents the multiband OFDM solution, followed by a description of our proposed SS-MC-MA scheme. Section III details the resource allocation algorithm applied to the SS-MC-MA scheme. Section IV describes the dynamic distribution of the timefrequency codes. Simulation results showing the interest of our proposed system in UWB applications are given in Section V before the conclusion in Section VI.

\section{SYSTEM MODEL}

\section{A. Multiband OFDM solution}

The MBOA solution for UWB systems design is based on the combination of an OFDM modulation with a multibanding approach, which divides the $7.5 \mathrm{GHz}$ UWB spectrum into 14 sub-bands of $528 \mathrm{MHz}$ each [7], as seen in Fig. 1. These subbands are then regrouped into 5 channels. Multibanding reduces power consumption and lowers cost since the information is processed over much smaller bandwidth, while taking benefit of the frequency diversity linked to the total bandwidth. Initially, most of the studies have been performed on the first 3 sub-bands $(3.1-4.8 \mathrm{GHz})$.

The multiband UWB system provides data rates ranging from 53.3 Mbps to $480 \mathrm{Mbps}$. The OFDM scheme consists of 128 subcarriers, out of which 100 are assigned to data tones. The modulation used is a quadrature phase-shift keying (QPSK), which leads to the transmission of 200 bits per OFDM symbol. The useful duration of each OFDM symbol is $242 \mathrm{~ns}$,

This work was supported by France Télécom R\&D/RESA/BWA, within the contract 46136582 . 
leading to a subcarrier frequency spacing of $4.125 \mathrm{MHz}$. A zero-padding cyclic prefix of duration $60.61 \mathrm{~ns}$ is added at the end of each OFDM symbol to cope with the inter-symbol interference (ISI). An additional guard interval of $9.47 \mathrm{~ns}$ is added for the sub-bands' hops.

The MBOA solution offers potential advantages for UWB applications, e.g., the signal robustness against channel selectivity and the efficient exploitation of the energy of every signal received within the prefix margin. However, this solution suffers from various limitations in a multi-user and multi-piconet context. When the first 3 sub-bands are considered, conflicts between users start as soon as we add a fourth user to the cell, whereas cells of up to 6 simultaneous users are being considered in the MBOA proposal. Moreover, this solution lacks in the ability to allocate sub-bands and power optimally since the sub-bands are not assigned to each user according to his channel condition, and the transmitted power is equally distributed among sub-bands without any power adaptation.

\section{B. Proposed SS-MC-MA scheme}

Some studies have proposed to use a MC-CDMA waveform by adding a CDMA scheme to the OFDM solution with a spreading in the frequency domain, in order to improve the system performance. The resource allocation becomes more flexible due to the addition of a spreading sequence. Furthermore, the spreading in the frequency domain improves the signal robustness against the frequency selectivity of the UWB channel, and against the narrowband interference, since the signal bandwidth could become much larger than the bandwidth of the interference.

To improve the system performance, we propose a SS-MCMA scheme [8]. SS-MC-MA consists in assigning to each user a specific block of subcarriers according to a frequency division multiple-access (FDMA) scheme, whereas with MCCDMA, resource sharing is realized by assigning to each user different individual codes transmitted over the whole available bandwidth. The SS-MC-MA system benefits from the performance and advantages of the MC-CDMA system while improving the resource allocation flexibility. With SS-MCMA, symbols are transmitted simultaneously on a specific subset of subcarriers by the same user and undergo the same distortions. Self-interference (SI), which replaces the multiple access interference (MAI) obtained with MC-CDMA, can be easily compensated by mono-user detection with only one complex coefficient per subcarrier.

The proposed SS-MC-MA scheme is applied to UWB while respecting the OFDM parameters of the MBOA solution in order not to increase the system complexity significantly. At a given time, each user is allocated a group of 100 subcarriers equivalent to one of the first $3 \mathrm{MBOA}$ sub-bands of $528 \mathrm{MHz}$ bandwidth. Each sub-band is then divided into several blocks $b$, each of them including a number of subcarriers equal to the spreading code length $L$ and linked by a spreading code $C_{b}$. The constellations used are the QPSK (similar to MBOA), as well as the 8-QAM and 16-QAM in order to better fit the channel capacity. Besides, the radio-frequency frond-end of the MBOA solution is maintained.

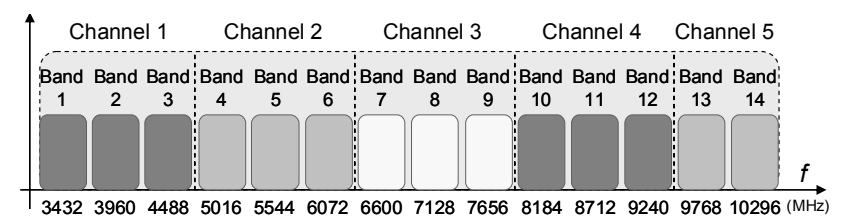

Figure 1. Sub-bands distribution of the MBOA system.

\section{Channel model}

The channel model used for our resource allocation is the one adopted by the IEEE 802.15.3a committee for the evaluation of UWB physical layer proposals [9]. It is a modified version of the Saleh-Valenzuela model for indoor channels [10], which fits the properties of measured UWB channels. A lognormal distribution is used for the multipath gain magnitude. In addition, independent fading is assumed for each cluster and each ray within the cluster. Moreover, four different channel models (CM1 to CM4) are defined for the UWB system modeling, each with arrival rates and decay factors chosen to match different usage scenarios and to fit line-of-sight (LOS) and non-line-of-sight (NLOS) cases.

\section{PROPOSED ALLOCATION ALGORITHM}

In this paper, we propose an efficient resource allocation algorithm for our proposed SS-MC-MA waveform, whose objective is to maximize the total system throughput with a PSD constraint fixed by the FCC regulations. The channel response varies slowly in time and can be considered as quasistatic during one frame. Hence, we can assume that the channel state information (CSI) is available at the transmitters.

The throughput in bits per symbol of an OFDM system is derived from Shannon theorem by

$$
R_{\text {OFDM }}=\sum_{k \in S} \log _{2}\left(1+\frac{1}{\Gamma}\left|H_{k}\right|^{2} \frac{P_{k}}{N_{0}}\right),
$$

where $S$ is the group of used subcarriers, $\Gamma$ the signal-to-noise ratio (SNR) gap, known as the normalized $S N R, H_{k}$ and $P_{k}$ the frequency-domain response and the transmitted power density of the $k^{\text {th }}$ subcarrier respectively, and $N_{0}$ the noise density.

The total throughput in bits per symbol of a SS-MC-MA system using a zero-forcing detection is given from [11] by

$$
R_{S S-M C-M A}=\sum_{b=1}^{B} \sum_{c=1}^{C_{b}} \log _{2}\left(1+\frac{1}{\Gamma} \frac{L^{2}}{\sum_{k \in S_{b}} \frac{1}{\left|H_{k}\right|^{2}}} \frac{P_{c, b}}{N_{0}}\right),
$$

where $B$ is the number of blocks, $C_{b}$ the number of codes per block $b, L$ the spreading code length, and $P_{c, b}$ the power density of code $c$ within block $b$.

The throughput maximization study is divided into two steps. First, we consider an infinite granularity case where the 
throughput $R$ is allowed to have real non-integer values, and then we consider a finite granularity case where the throughput values must be integer since we have discrete modulation orders. In the infinite granularity case, by imposing a constraint on the PSD, the optimization problem can be described as

$$
\max \sum_{b=1}^{B} \sum_{c=1}^{C_{b}} \log _{2}\left(1+\frac{1}{\Gamma} \frac{L^{2}}{\sum_{k \in S_{b}} \frac{1}{\left|H_{k}\right|^{2}}} \frac{P_{c, b}}{N_{0}}\right),
$$

subject to

$$
\sum_{c=1}^{C_{b}} P_{c, b} \leq P \quad \text { and } \quad P_{c, b} \geq 0 \quad \forall b,
$$

with $P$ the PSD constraint of $-41.25 \mathrm{dBm} / \mathrm{MHz}$. To maximize the system throughput for a given PSD, the $L$ subcarriers with the largest response amplitude should be selected.

To solve the optimization problem, we introduce the Lagrange multipliers $\lambda$ in (3) and (4). We find that

$$
P_{c, b}=P / C \quad \text { and } \quad C=L .
$$

Consequently, the maximum reachable throughput in bits per block $b$ in the infinite granularity case becomes

$$
R_{b}^{*}=L \log _{2}\left(1+\frac{1}{\Gamma} \frac{L}{\sum_{k \in S_{b}} \frac{1}{\left|H_{k}\right|^{2}}} \frac{P}{N_{0}}\right),
$$

and the maximum reachable throughput in bits per code $c$ becomes

$$
R_{c, b}^{*}=\frac{R_{b}^{*}}{L} \quad \forall c \in[1: L] .
$$

Since we have discrete modulation orders, the throughput per code must always have integer values. An optimal solution for this problem is given in [11] and it shows that an optimal throughput distribution would be to allocate $\left\lfloor R_{b}^{*} / L\right\rfloor+1$ bits to $n$ codes and $\left\lfloor R_{b}^{*} / L\right\rfloor$ bits to the remaining $L-n$ codes, where $n$ is an integer number given by

$$
n=\left\lfloor L\left(2^{R_{b}^{*} / L-\left\lfloor R_{b}^{*} / L\right\rfloor}-1\right)\right\rfloor .
$$

Hence, the discrete throughput in bits per code becomes

$$
\begin{cases}\bar{R}_{c, b}=\left\lfloor R_{b}^{*} / L\right\rfloor+1 & \forall c \in[1: n] \\ \bar{R}_{c, b}=\left\lfloor R_{b}^{*} / L\right\rfloor & \forall c \in[n+1: L] .\end{cases}
$$

The power density per code is then given by

$$
\bar{P}_{c, b}=\frac{\Gamma}{L^{2}} \sum_{k \in S_{b}} \frac{N_{0}}{\left|H_{k}\right|^{2}}\left(2^{\bar{R}_{c, b}}-1\right),
$$

and the total discrete throughput in bits per block $b$ becomes

$$
\begin{aligned}
\bar{R}_{b}=\left(\left\lfloor L\left(2^{R_{b}^{*} / L-\left\lfloor R_{b}^{*} / L\right\rfloor}-1\right)\right\rfloor\right)\left(\left\lfloor R_{b}^{*} / L\right\rfloor+1\right) \\
+\left(L-\left\lfloor L\left(2^{R_{b}^{*} / L-\left\lfloor R_{b}^{*} / L\right\rfloor}-1\right)\right\rfloor\right)\left(\left\lfloor R_{b}^{*} / L\right\rfloor\right) .
\end{aligned}
$$

\section{TIME-FREQUENCY CODES EXPLOITATION}

\section{A. TFC distribution}

In the MBOA proposal, unique logical channels corresponding to different piconets are defined by using different time-frequency codes (TFC) for each band group. These codes provide frequency hopping from a band to another at the end of each OFDM symbol. Fig. 2 represents a realization of the TFC, where the first OFDM symbol of the first user is transmitted on Band 1, the second symbol on Band 2 and the third on Band 3. The proposition described by the MBOA norm consists in choosing the TFC regularly without taking into consideration the channel response state of each user for each of the first $3 \mathrm{MBOA}$ sub-bands. In our study, we use a dynamic TFC (DTFC) distribution, different from the distribution of the MBOA norm, which consists in selecting for every 3 consecutive OFDM symbols the optimal repartition of the 3 users on the 3 sub-bands that maximizes the SS-MC-MA system margin.

We consider 3 users, and consequently 3 TFC codes, distributed on the first 3 bands of the MBOA solution. Over a given OFDM symbol period $T$, each user occupies one of the 3 available sub-bands. To find the number of unique possible distributions of the TFC over 3 consecutive periods $T$, we have a combinational problem. In fact, over one period, we have a permutation with order and without repetition of 3 users on 3 bands. The total number of possible permutations is then given by $p=n ! /(n-k) !=6$, with $n=3$ the number of bands and $k=3$ the number of users. Hence, there are 216 possible permutations over 3 periods. But within each band, we do not take into consideration the users' order in time, thus the number of unique permutations is reduced to 55 . We should notice that we have a total of 9 different channel responses in the system, since each user has 3 different channel responses corresponding to 3 bands.

\section{B. Algorithm implementation}

After having described the bit loading algorithm of the proposed SS-MC-MA scheme and the dynamic TFC distribution for system throughput maximization, we combine these 2 techniques into a single algorithm and we apply it to the first 3 sub-bands of the MBOA solution. The total number of blocks per sub-band is equal to $\lfloor N / L\rfloor$, where $N=100$ is the number of used subcarriers. This algorithm can be implemented as follows:

1) Initialization

Set $\quad R_{\text {code }, \text { block }}=0$ and $P_{\text {code }, \text { block }}=0, \forall$ code, block, 


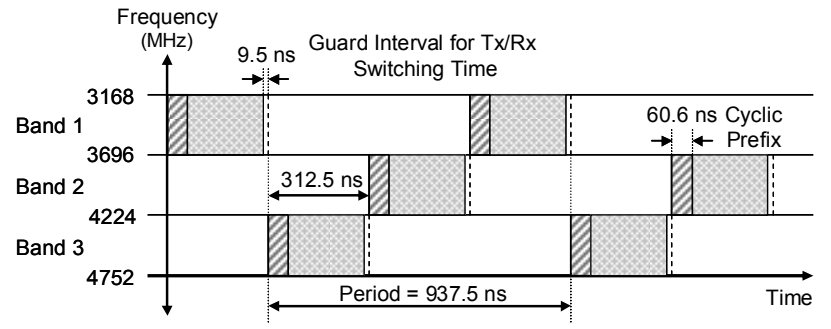

Figure 2. TFC realization for the MBOA solution.

$S_{\text {block }}=\phi, \forall$ block,

$L=$ spreading code length,

$N=$ number of data subcarriers,

$H_{i, j}=$ channel response of user $i$ on band $j$,

Combin $=\left\{\right.$ Comb $_{1} \ldots$ Comb $\left._{55}\right\}=$ TFC combinations.

2) For $i=1$ to 3 and $j=1$ to 3 ,

for block $=1$ to $\lfloor N / L\rfloor$,

find $S_{\text {block }}=$ best $L$ subcarriers in $H_{i, j}$,

update $H_{i, j}: H_{i, j}=H_{i, j}-S_{\text {block }}$,

find the block throughput $R_{b}^{*}$ from (6),

compute the code throughput,

$$
\begin{gathered}
\text { for code }=1 \text { to } n, \\
R_{\text {code } \text { block }}=\left\lfloor R_{b}^{*} / L\right\rfloor+1, \\
\text { for code }=n+1 \text { to } L, \\
R_{\text {code }, \text { block }}=\left\lfloor R_{b}^{*} / L\right\rfloor, \\
\text { with } n \text { given from (8), }
\end{gathered}
$$

symbol throughput: $\bar{R}_{\text {symbol }}=\sum_{\text {code, block }} R_{\text {code }, \text { block }}$.

3) For Combin $=\mathrm{Comb}_{1}$ to $\mathrm{Comb}_{55}$,

compute the system throughput over 3 symbols with 3 users,

$$
\begin{gathered}
R_{\text {syst }}(\text { Combin })=\sum_{\text {Combin }} \bar{R}_{\text {symbol }}(\text { Combin }), \\
\text { OptThroughput }=\max \left(R_{\text {syst }}\right), \\
\text { OptTFC }=\text { Combin }(\text { OptThroughput }) .
\end{gathered}
$$

4) Output

Return OptThroughput and OptTFC.

\section{Simulation ReSUlts}

In this section, we present the simulations performed on the first 3 sub-bands of the MBOA solution using the proposed adaptive SS-MC-MA system, with and without the dynamic TFC (DTFC). The spreading code length used is the optimal one with $L=16$, which gives the highest system throughput. First, the algorithm is used to maximize the total system throughput. The simulations are performed by considering the case of a PSD constraint per one OFDM symbol (Fig. 3), and the case of a mean PSD constraint per three OFDM symbols where the PSD measuring is made on 3 symbols (Fig. 4). In the second case, if a user is occupying a sub-band for only onethird of the time, his PSD could be 3 times larger than the average PSD defined by the MBOA norm $(-41.25 \mathrm{dBm} / \mathrm{MHz})$.
Fig. 3 represents the total system throughput per 3 OFDM symbols for different channel attenuation levels, with a 4-QAM modulation. With the MBOA system using CM2 channel model, the 3 users are able to transmit information $(3 \times 3 \times 200=1800 \mathrm{bit} / 3 \times$ symbol $)$ at attenuation levels lower than $31 \mathrm{~dB}$. At attenuation levels from 32 to $35 \mathrm{~dB}$, only 2 users are able to transmit (1200 bit/3×symbol), at levels from 36 to $41 \mathrm{~dB}$ only 1 user is able to transmit ( $600 \mathrm{bit} / 3 \times$ symbol), and at levels higher than $46 \mathrm{~dB}$ no one is able to transmit. When we consider an adaptive SS-MC-MA system, the throughputs are much higher at high attenuation levels, e.g. the throughput of $1200 \mathrm{bit} / 3 \times$ symbol is reached at $35 \mathrm{~dB}$ level with MBOA, whereas with SS-MC-MA it can be reached at $71 \mathrm{~dB}$, and at $35 \mathrm{~dB}$ the system has a throughput of 1800 bit $/ 3 \times$ symbol. Moreover, the system is able to transmit at an attenuation level of $93 \mathrm{~dB}$. Besides, when we apply the TFC distribution algorithm to the adaptive SS-MC-MA system, the throughput increases more as we can see in Fig. 3.

From Fig. 4, we notice that even with a mean PSD constraint per 3 symbols, the adaptive SS-MC-MA system outperforms MBOA, and when we use the DTFC distribution, the total throughput increases more. Similar results are obtained with the different channel models (CM1-CM4). Fig. 5 represents an adaptive SS-MC-MA system using a 16-QAM modulation. This system also outperforms MBOA and is able to transmit data at an attenuation level of $92 \mathrm{~dB}$.

This algorithm is also used to maximize the throughput of the user which has the most critical channel response. Fig. 6 gives the throughput of the critical user for CM2 and CM4 model. We notice that with MBOA using CM2 model, this user is only able to transmit information at attenuation levels lower than $31 \mathrm{~dB}$ whereas with adaptive SS-MC-MA, he can even transmit at $92 \mathrm{~dB}$.

\section{CONCLUSION}

In this paper, we proposed a low-complexity resource allocation algorithm applied to a SS-MC-MA waveform which is new in high data rate UWB applications, and which respects the OFDM parameters of the MBOA solution in order not to increase the system complexity significantly. The proposed system aims at maximizing the total throughput while taking into consideration the WPAN environment and respecting the FCC regulations. Furthermore, we proposed a dynamic distribution of the time-frequency codes which increases the system performance since with the MBOA solution the TFC are distributed regularly without taking into consideration the channel response state of each user for each band. We found that our proposed system outperforms the MBOA system and transmits information at much higher attenuation levels with higher throughputs. Hence, we conclude that our proposed system can be advantageously exploited for high data rate UWB applications.

\section{REFERENCES}

[1] "First report and order, revision of part 15 of the commission's rules regarding ultra-wideband transmission systems," FCC, ET Docket 98153, Feb. 14, 2002.

[2] IEEE 802.15 WPAN High Rate Alternative PHY Task Group 3a (TG3a). Available: www.ieee802.org/15/pub/TG3a.html. 


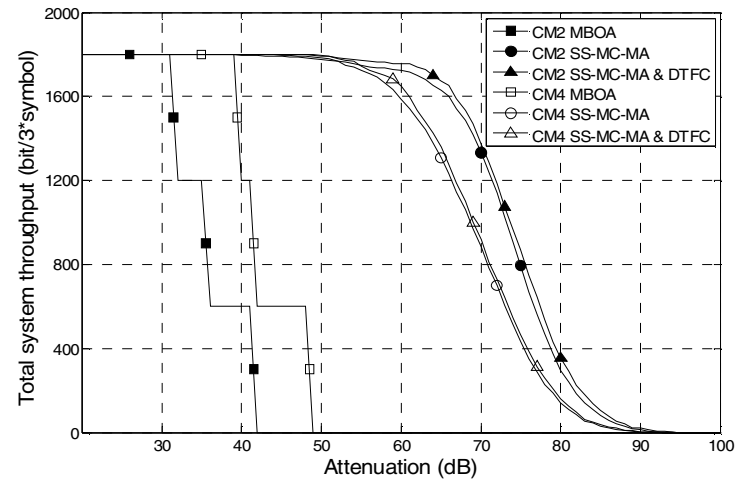

Figure 3. Total system throughput with a 4-QAM in the case of a PSD constraint per OFDM symbol for CM2 and CM4 channel models.

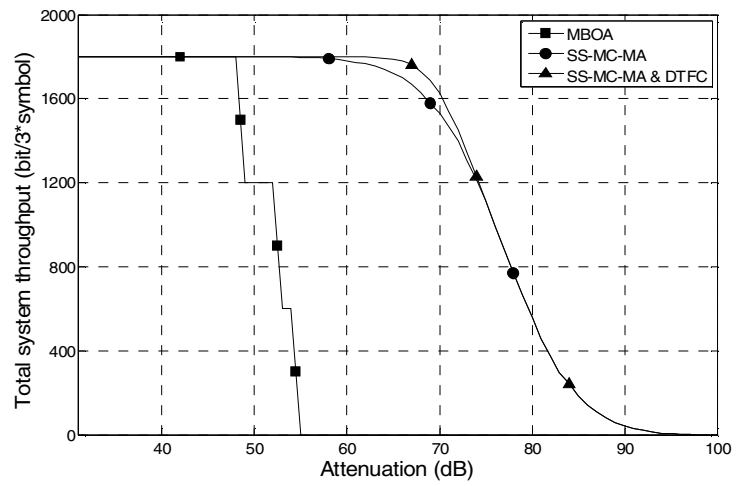

Figure 4. Total system throughput with a 4-QAM in the case of a mean PSD constraint per 3 OFDM symbols for CM1 channel model.

[3] IEEE P802.15 Working Group for Wireless Personal Area Networks (WPANs), "Multi-band OFDM physical layer proposal for IEEE 802.15 task group 3a," Sept. 2004.

[4] W. Tung and J. Wang, "MMSE receiver for multicarrier CDMA overlay in ultra-wide-band communications," IEEE Trans. on Vehicular Technology, vol. 54, no. 2, pp. 603-614, March 2005.

[5] Z. Xu and L. Liu, "Power allocation for multi-band OFDM UWB communication networks," in Proc. IEEE Vehicular Technology Conference, vol. 1, pp. 368-372, Sept. 2004.

[6] A. Stephan, J-Y. Baudais and J-F. Hélard, "Resource allocation for multicarrier CDMA systems in ultra-wideband communications," in Proc. IEEE International Telecommunications Symposium (ITS2006), pp. 1002-1007, Fortaleza, Brazil, Sept. 2006.

[7] A. Batra et al., "TI physical layer proposal for IEEE 802.15 task group 3a," IEEE P802.15-03/142r2-TG3a, Mar. 2003.

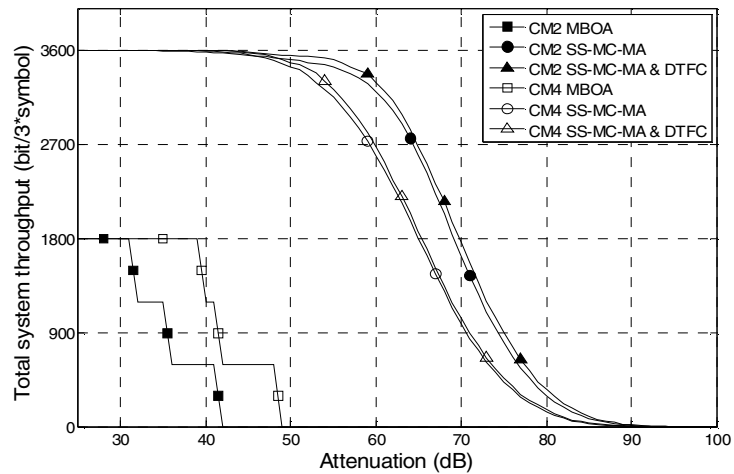

Figure 5. Total system throughput with a 16-QAM in the case of a PSD constraint per OFDM symbol for CM2 and CM4 channel models.

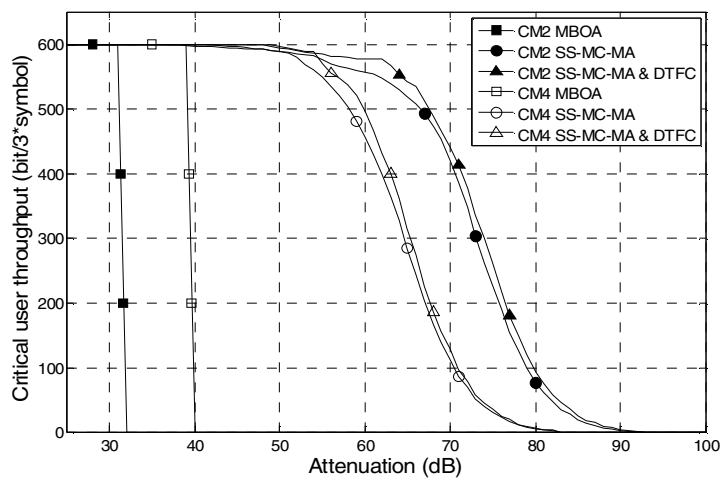

Figure 6. Critical user throughput with a 4-QAM in the case of a PSD constraint per OFDM symbol for CM2 and CM4 channel models.

[8] S. Kaiser and K. Fazel, "A flexible spread-spectrum multi-carrier multiple-access system for multi-media applications," in Proc. IEEE Intern. Symp. on Personal, Indoor and Mobile Radio Commun. (PIMRC'97), pp. 100-104, Helsinki, Finland, Sept. 1997.

[9] J. Foerster et al., "Channel modeling sub-committee report final," IEEE802.15-02/490, Nov. 2003.

[10] A. Saleh and R. Valenzuela, "A statistical model for indoor multipath propagation," IEEE J. on Selected Areas in Commun., vol. 5, no. 2, pp. 128-137, Feb. 1987.

[11] M. Crussière, J-Y. Baudais and J-F. Hélard, "Adaptive spread spectrum multicarrier multiple access over wirelines," IEEE J. on Selected Areas in Commun., Special Issue on Power Line Commun., vol. 24, no. 7, pp. 1377-1388, July 2006. 